\title{
The Influence of Emotional State and Pictorial Cues on Perceptual Judgments
}

\section{Human Factors and Ergonomic Society}

\author{
Kimberly R. Raddatz \\ Abigail Werth \\ Tuan Q. Tran
}

\section{October 2007}

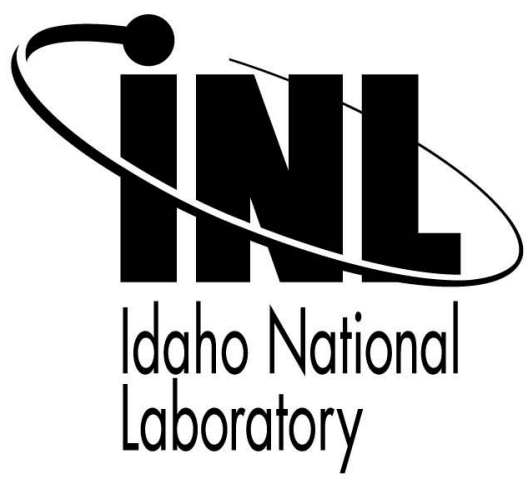

This is a preprint of a paper intended for publication in a journal or proceedings. Since changes may be made before publication, this preprint should not be cited or reproduced without permission of the author. This document was prepared as an account of work sponsored by an agency of the United States Government. Neither the United States Government nor any agency thereof, or any of their employees, makes any warranty, expressed or implied, or assumes any legal liability or responsibility for any third party's use, or the results of such use, of any information, apparatus, product or process disclosed in this report, or represents that its use by such third party would not infringe privately owned rights. The views expressed in this paper are not necessarily those of the United States Government or the sponsoring agency. 


\title{
The Influence of Emotional State and Pictorial Cues on Perceptual Judgments
}

\author{
Kimberly R. Raddatz ${ }^{1}$, Abigail Werth ${ }^{1}, \&$ Tuan Q. Tran ${ }^{2}$ \\ ${ }^{1}$ Kansas State University, Manhattan, KS \\ and \\ ${ }^{2}$ Idaho National Laboratory, Idaho Falls, ID
}

\begin{abstract}
Perspective displays (e.g., CDTI) are commonly used as decision aids in environments characterized by periods of high emotional arousal (e.g., terrain enhanced primary flight displays). However, little attention has been devoted to understanding how emotional state, independently or in conjunction with other perceptual factors (e.g., pictorial depth cues), can impact perceptual judgments. Preliminary research suggests that induced emotional state (positive or negative) adversely impacts size comparisons in perspective displays (Tran \& Raddatz, 2006). This study further investigated how size comparisons are affected by emotional state and pictorial depth cues while attenuating the limitations of the Tran \& Raddatz (2006) study. Results confirmed that observers do make slower judgments under induced emotional state. However, observers under negative emotional state showed higher sensitivity (d') and required more evidence to respond that a size difference exists (response bias) than observers under positive emotional state. Implications for display design and human performance are discussed.
\end{abstract}

\section{INTRODUCTION}

Human factors considerations are integral to the design and use of perspective displays considering that these displays often must support judgment and decision-making in environments where misperceptions have severe consequences. For example, terrain-enhanced primary flight displays present perspective views of terrain that are designed to facilitate pilots' avoidance of controlled flight into terrain (CFIT) accidents. Cockpit displays of traffic information (CDTIs) shown in perspective are designed to enhance pilots' ability to avoid traffic collisions. In these examples, the accurate scaling of size across projected depth is the precursor to the spatial judgments that must be made to maintain safety. Therefore, it is important to understand the role of pictorial depth cues in the support of veridical size scaling.

Furthermore, when pilots are facing and trying to avoid traffic or terrain collisions, they are often doing so under increased stress, workload, and consequently, emotional arousal. Therefore it is also important to consider the influence of arousal and emotional state on performance effectiveness. This study was designed to investigate whether emotional state interacts with specific pictorial cues in the support of size scaling.

The relationship between emotional arousal (i.e., physiological experience) and emotional state (i.e., psychological experience) on human performance is still uncertain. According to the capacity-resources hypothesis, general arousal (independent of emotion state) can drain attentional resources and decrease performance (Kahneman, 1973). Emotional state (positive and negative) has also been shown to affect human performance in different ways (e.g., Clore, Schwarz, \& Conway, 1994). For example, Isen (2000) demonstrated the cognitive benefits of positive emotional state (e.g., better memory, flexibility, creativity, more efficient decision-making strategies). However, Clore et al. (1994) concluded that a positive emotional state induces more surface processing, leading to the use of heuristics in decision-making while negative emotional state induces more detailed and systematic processing, leading to better decision-making.

Such inconsistencies in the literature led to an initial investigation of the relationship between emotional states and perspective size judgments by Tran and Raddatz (2006). The size judgment task was studied both because of its importance to perception in perspective displays and because of the existing literature on how size judgments are affected by effortful processing (e.g., Carlson, 1960). Tran and Raddatz (2006) had participants make size comparisons under conditions of induced emotional states (positive, negative, neutral) in a dual-task paradigm. Results showed that participants in both the positive and negative induced emotional states were slower to make accurate size judgments than those not under an induced emotional state. These results supported Kahneman's (1973) capacity-resources hypothesis and provided a preliminary indication that emotional arousal independent of valence can influence visual performance on perspective displays. However, the dual task complexity of the study resulted in high error rates for size comparisons so implications were more speculative in nature. Therefore, this study was designed to replicate and clarify the results of Tran and Raddatz using a simple size comparison task in a single task environment.

The present study was also designed to investigate whether emotional state affects individuals' response bias. Past research suggests that individuals experiencing negative emotion expend more time and effort acquiring additional information to assess a situation than individuals experiencing positive emotion (Staal, 2006). Restated within a signal detection theory (SDT) framework, negative emotion may induce a more conservative response bias (i.e., observers require more information before making a response) while positive emotion may induce a more liberal response bias (i.e., observers require less information before making a response). Also of interest is whether the presence of more depth cues interacts with emotional state to systematically influence response bias. 


\section{METHOD}

\section{Participants}

Fifty-three general psychology student observers participated in the experiment for class credit and were randomly assigned to one of the three emotion conditions. Data from eleven observers were discarded due to below chance accuracy, failure of the emotion manipulation, and/or equipment failure. The remaining 42 observers experienced the negative (15), positive (12) or neutral (15) emotion conditions.

\section{Materials}

Emotional state was manipulated through the use of short film clips. Observers in the positive emotion condition saw a scene from "When Harry Met Sally" depicting two adults in a restaurant discussing whether or not women can fake sexual excitement. Observers in the negative emotion condition saw a scene from "The Champ" where a child tearfully witnesses his father fatal injuries from a boxing match. Observers in the neutral condition saw a scene from "Shapes," simulating a screen saver by showing colorful geometric shapes. All film clips lasted approximately $2 \frac{1}{2}$ minutes and were previously validated as successful elicitations of their respective emotional state (Gross \& Levenson, 1995).

Assessment of Emotion Manipulation. An emotion scale adapted from the Brief Mood Introspection Scale (BMIS; Niedenthal et al., 1997) was used to measure observers' emotion. The BMIS lists 16 affect adjectives (i.e., lively, happy, sad, tired, caring, content, gloomy, jittery, drowsy, grouchy, peppy, nervous, calm, loving, fed up, and active) embedded in sentences with the following stem: "I presently feel _. . "Observers were instructed to indicate the degree to which they were presently feeling each statement on a 5-point Likert scale with "1" indicating "definitely do not feel," and " 5 " indicating "definitely do feel." The BMIS has shown good validity and reliability properties in the literature (see Mayer \& Gaschke, 1988 for details).

Size Judgment Stimuli. Using commercial terrain generating software (World Construction Set, 1998), perspective displays were created with two different types of terrain (reduced cue and rectilinear) upon which two red blocks were placed (See Figures 1 and 2). Both terrains had the important depth cues of aspect ratio of the blocks and height in plane. The rectilinear terrain had the added cues of linear perspective and foreshortening. The relative distance difference between the blocks in projected depth varied $(0,3$, 6 or 9 units) as did the size ratio of the block widths $(1: 1,2: 3$, $2: 1,3: 1)$. A distance difference of 0 indicated blocks were placed at the same depth, meaning that blocks of equal proximal width (i.e., width on the screen) had equal distal width. Distance differences greater than 0 indicated that the effects of depth on the proximal images must be attenuated, meaning that blocks of equal proximal width did not have equal distal width. Size ratio of 1:1 indicated that the blocks had the same distal width. Size ratio of 3:1 indicated that the distal width of one block was three times the distal width of the other block. Observers were asked to compare distal block widths using phenomenal or "first-look" instructions (e.g., Carlson, 1960). The distance difference and size ratio variables combined to vary the difficulty of the comparison task such that smaller size ratios and larger distance differences were more difficult to judge.

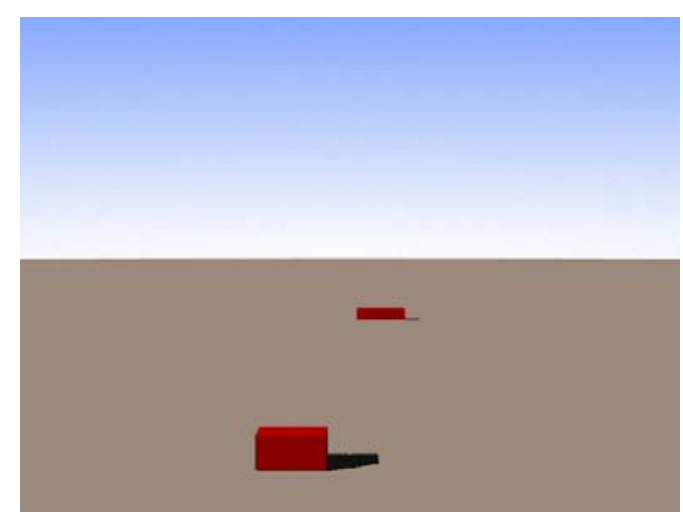

Figure 1. Example of the reduced-cue terrain condition (distance difference $=2$, size ratio $=2: 1$ ).

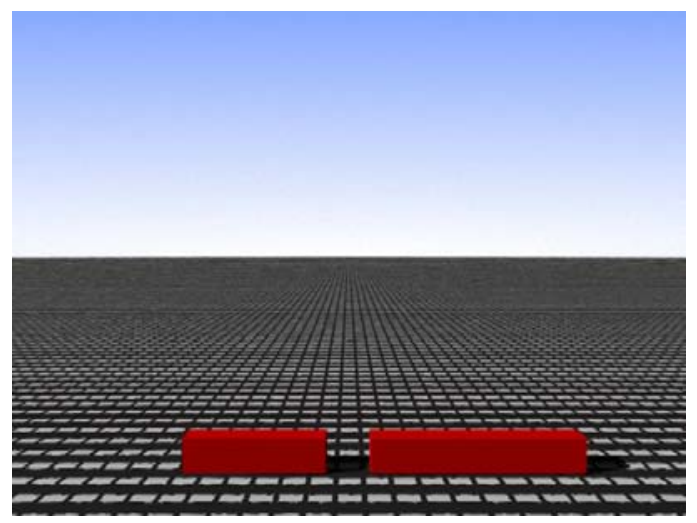

Figure 2. Example of the rectilinear terrain condition (distance difference $=0$, size ratio $=3: 2$ )

Filler Tasks. Two tasks, the asterisk task and the affective picture task, served to reduce proactive interference of the size judgment stimuli. The asterisk task, given on onethird of the trials, consisted of a screen displaying 10-30 asterisks in various colors. Observers were given $2 \mathrm{~s}$ to count the number of red asterisks and enter that number via the computer keyboard. The pictures in the affective picture task, given on two-thirds of the trials, were taken from the International Affective Picture System (IAPS) database (Lang, Bradley, \& Cuthbert, 1997). The IAPS database consists of over 1000 standardized affect-laden pictures that have been rated on three dimensions: valence, arousal, and dominance. All pictures used in the affective picture task were controlled for valence: positive pictures had the highest mean valence (7.56), followed by neutral pictures (5.57), and negative pictures (3.89). The picture valence was congruent with the participant's emotion condition (e.g., only positive pictures were presented in positive emotion condition, negative 
pictures in the negative emotion condition, neutral pictures in the neutral emotion condition). Observers viewed each picture for $2 \mathrm{~s}$ and rated the degree of positive or negative valence using a 7-point scale with "1" indicating "not at all" to "7" indicating "extremely" negative/positive. In addition to reducing proactive interference, the picture task was also meant to maintain or enhance the induced emotional state.

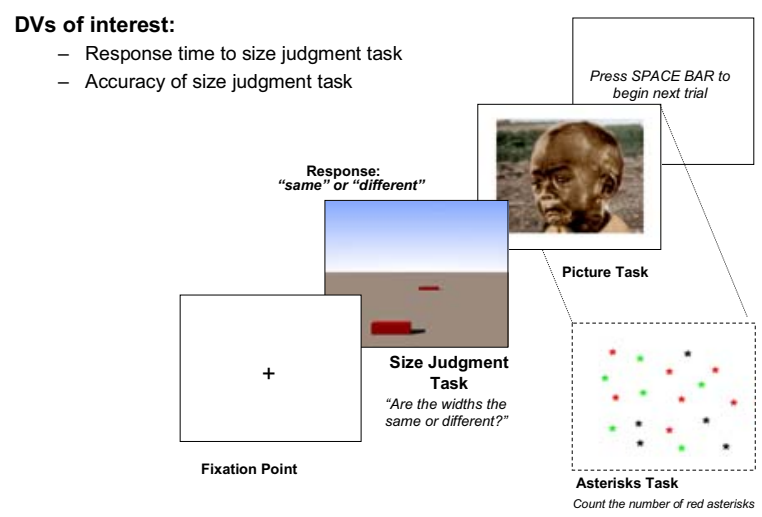

Figure 3. Depiction of a trial procedure.

\section{Design and Procedure}

The proposed study was a 3(Emotional State: positive, negative, neutral) x 2(Terrain: reduced cue, rectilinear) x 4 (Distance Difference: 0, 3, 6, 9 units) x 4 (Size Ratio: 1:1, 3:2, 2:1, 3:1) mixed design with emotional state as the between-subjects variable. For the current study, only the variables of emotional state and terrain were analyzed.

Each trial began with a fixation point presented for $500 \mathrm{~ms}$ followed by the size judgment task (Figure 3 ). Observers selected the key labeled "=" if they perceived the distal widths of the blocks to be the same and the key labeled " $D$ " if they perceived a difference. They then viewed and responded appropriately to either the picture or the asterisk task.

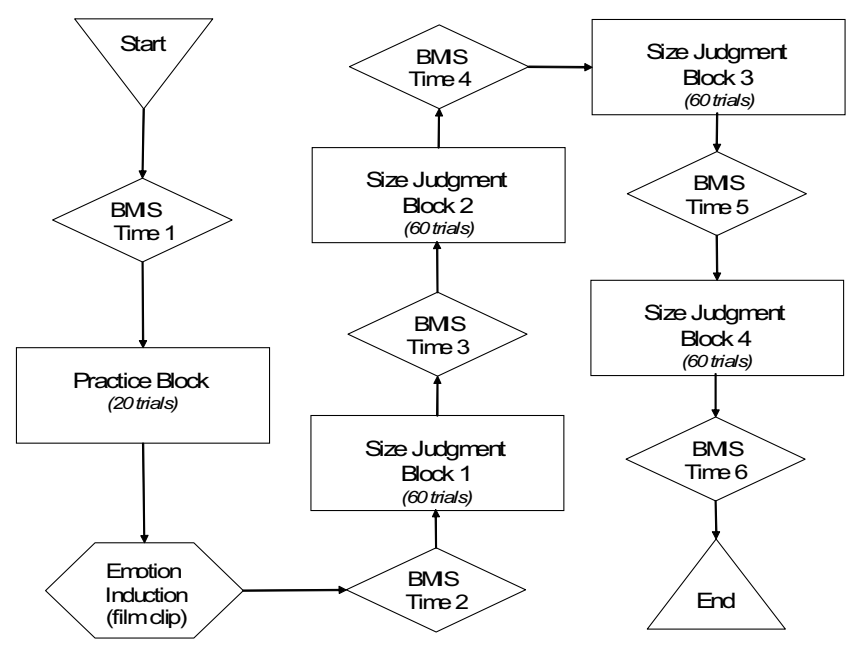

Observers began by completing the BMIS to provide a baseline measurement of their current emotional state (see Figure 4). They then completed a block of 20 practice trials, followed by the movie clip and another BMIS. Observers then completed 4 counterbalanced blocks of testing, with each block consisting of sixty trials of the same terrain type. At the end of each block, observers completed a BMIS. Observers participated in groups of 1, 2, or 3. E-Prime (Schneider, Eschman, \& Zuccolotto, 2002) was used to administer the trials and record observers' responses.

\section{RESULTS}

\section{Emotion Assessment Scores}

Independent t-tests were conducted to verify the emotion manipulation. Results revealed that observers in the positive emotion condition had significantly higher positive BMIS scores than observers in the negative emotion condition from Time 2 to Time 6 ( $\left.\mathrm{p}^{\prime} \mathrm{s}<.05\right)$. Observers in the negative emotion condition had significantly higher negative BMIS scores than observers in the positive emotion condition from Time 2 to Time 5 (p's<.05) but only marginally higher at Time $6(\mathrm{p}=.08)$ (Figures 5 and 6). Overall, the manipulation was successful for both the positive and negative emotion conditions.

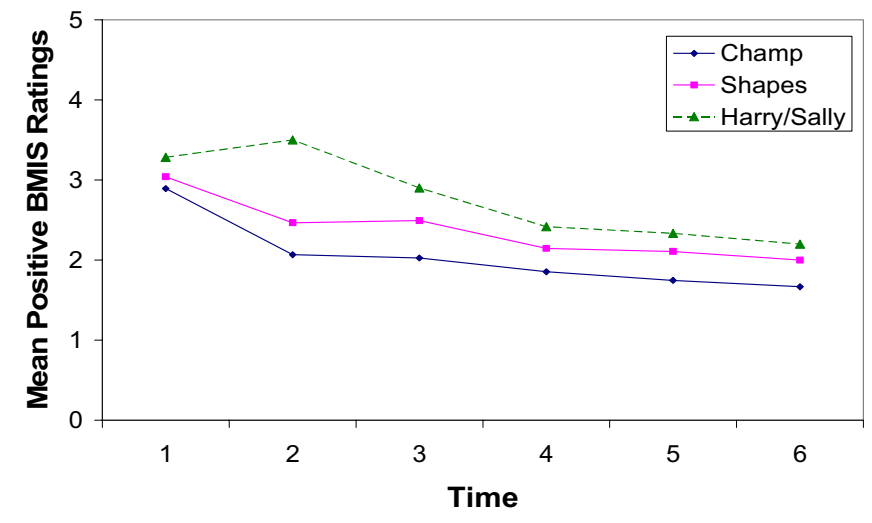

Figure 5. Positive BMIS results.

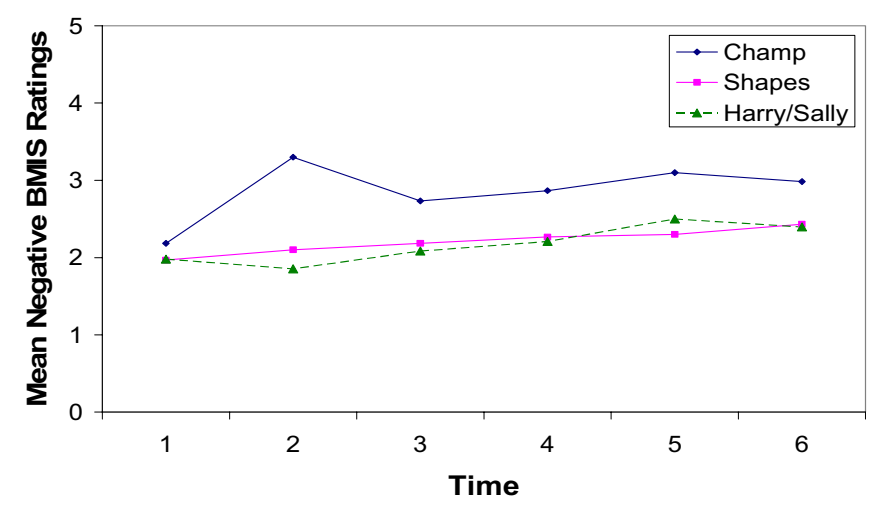

Figure 6. Negative BMIS results.

Figure 4. Depiction of the experimental procedure. 


\section{Response Time Analysis}

Consistent with Tran and Raddatz (2006), observers were slower to make size comparisons under an induced emotional state (Figure 7). This result supports Kahneman's capacity-resources hypothesis suggesting that general arousal drains resources, resulting in increased response time. Further, observers under negative and neutral emotion took more time to make their judgments in the presence of more depth information (i.e., the rectilinear terrain) while observers in the positive condition took less time in the presence of more depth information (Figure 8). This result suggests that observers under positive emotion were, indeed, engaged in more surface-level processing and therefore did not spend extra time processing the additional information while observers in negative and neutral emotion were more systematic in their processing, and thus took time to evaluate the additional information before making their decision.

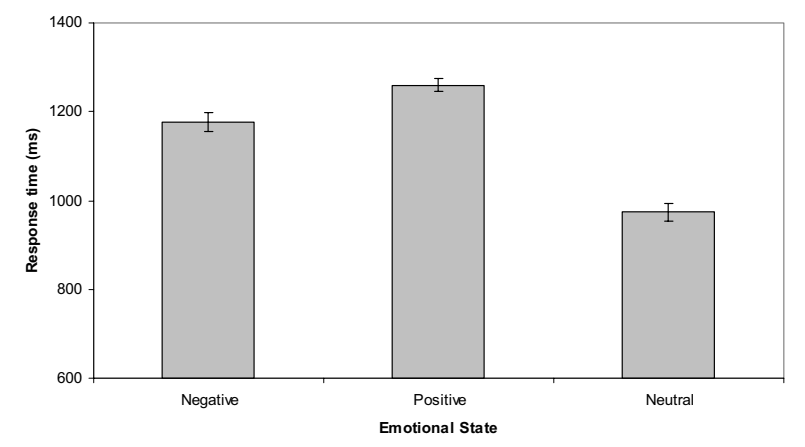

Figure 7. Response time for emotional state.

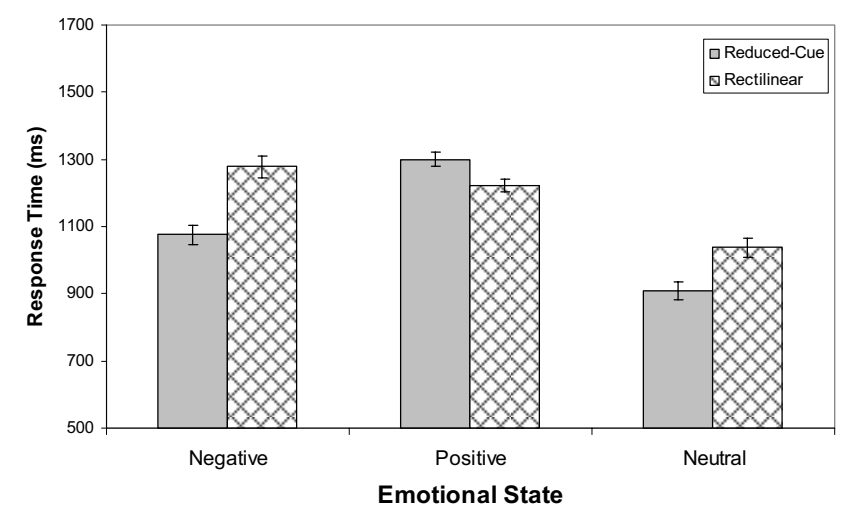

Figure 8. Response time for emotional state $\mathrm{x}$ terrain condition.

\section{SDT: Sensitivity and Response Bias}

A signal detection analysis allowed for the identification of factors that differentially influence perceptual ability to perceive a size difference (d') and the decision criterion for responding (c) (MacMillan \& Creelman, 1991). A size difference was defined as a "signal." Observers under positive emotion were less sensitive to the presence of a size difference than those under negative and neutral emotion. Further, the presence of more depth cue information in the rectilinear terrain only affected sensitivity of observers under positive induced emotion (Figure 9). Two informational cues to size comparison, height in plane and aspect ratio, were present in both terrain conditions. Thus, the additional texture provided by linear perspective and foreshortening was not necessary to support the size judgments. The fact that terrain only affected sensitivity under positive emotion suggests that observers under positive emotion are less sensitive to the relevant cues for detection. In other words, observers in the negative condition may have been engaged in more systematic processing that allowed them to focus on the more relevant cues while the surface level processing engaged in by observers in positive emotion left them less able to perceptually identify the relevant information.

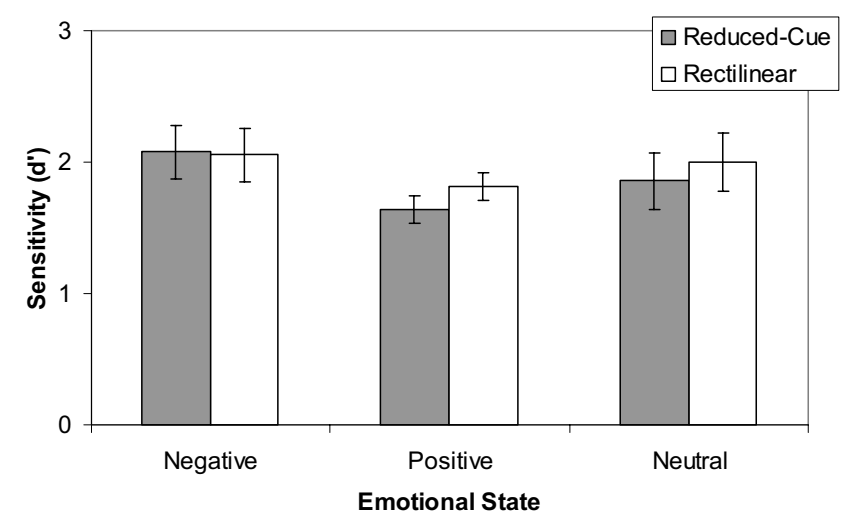

Figure 9. Sensitivity for emotional state $\mathrm{x}$ terrain condition.

Overall, observers adopted a bias toward responding that a size difference exists, which is consistent with previous research (e.g., Raddatz, Uhlarik, \& Foura, 2004). However, observers under negative emotion adopted a more conservative response bias relative to the other two emotion conditions. Thus, relative to observers in the other emotion conditions, observers under negative emotion required more information that size difference existed before responding accordingly (Figure 10). This finding is consistent with previous research suggesting that individuals under negative emotion engage in more thorough, systematic processing while making judgments (Staal, 2006) which, in this case, translated into the adoption of a more conservative response criterion.

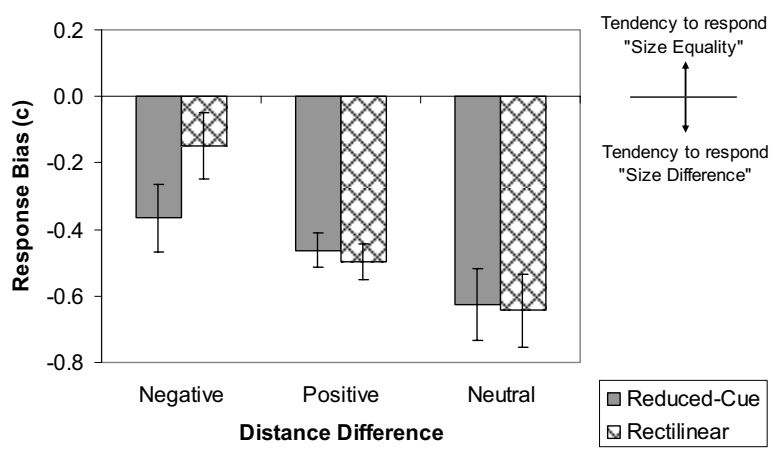

Figure 10. Effect of terrain condition and emotional state on response bias. 


\section{DISCUSSION}

The results of this study confirm that emotional arousal, regardless of valence, has at least some detrimental effect on decision-making in a size comparison task. Observers under no induced emotion showed the highest sensitivity with the quickest response time. However, important decisions in the real world cannot always be made in a neutral emotional state. This is especially true of judgments that must be made in such dynamic environments as airplane cockpits or nuclear control rooms. For example, emotional arousal often increases in situations in which a pilot is flying and suddenly the terrain awareness warning system (TAWS) alarm sounds. Wickens et al. (2004) characterized a nuclear power plant operator's environment as "hours of intolerable boredom punctuated by a few minutes of pure hell" (p. 508). For instance, during the Three-Mile Island incident, more than 100 alarms were initiated in the control room. Decisions made in these types of situations must be quick and accurate while under an induced emotional state.

The results of this study suggest that individuals, when under an induced emotional state, exhibit better decision-making under negative rather than positive emotion. Relative to positive emotion, observers under negative emotion exhibit higher sensitivity, quicker response times, and less response bias (requiring more evidence that a signal exists before responding accordingly). Also, observers under negative emotion exhibited high sensitivity regardless of the number of depth cues present which suggests they were better able to identify the important cues on which base their decisions.

\section{IMPLICATIONS}

The results of this study have implications beyond the domain of perspective displays. For instance, the area of augmented cognition is focused on developing computational methods using physiological sensors (e.g., eye-tracking, galvanic skin response, inter-heart beat) to identify information processing bottlenecks inherent in humancomputer interaction (Schmorrow, Stanney, Wilson, \& Young, 2006). Currently, level of arousal is used as the primary source of input to assess operator's functional state. Similarly, the human reliability community uses general arousal/stress as a major factor in their human error probability equation to assess human risk (Boring et al., 2007). The results of the proposed study suggest that these methods need to develop more precise sensors or equations to discriminate positive and negative emotional states for more accurate assessment of human performance.

\section{ACKNOWLEDGEMENTS}

This research was conducted while the third author was a doctoral student at Kansas State University.

\section{REFERENCES}

Boring, R.L., Whaley, A.M., Tran, T.Q., McCabe, P.H., Blackwood, L.G., \& Buell, R.F. (2007). Guidance on performance shaping factors assignment in SPAR-H (INL/EXT-06-11959, Draft). A Letter Report Prepared by the Idaho National Laboratory for the United States Nuclear Regulatory Commission.

Carlson, V.R. (1960). Overestimation in size-constancy judgments. American Journal of Experimental Psychology, 63, 199-213.

Clore, G. L., Schwarz, N., \& Conway, M. (1994). Affective causes and consequences of social information processing. In R. S. Wyer \& T. K. Srull (Eds.), Handbook of social cognition ( $2^{\text {nd }}$ Ed., Vol. 1, pp. 323-418). Hillsdale, NJ: Lawrence Erlbaum Associates, Inc.

Gross, J. \& Levenson, R. (1995). Emotion elicitation using films. Cognition and Emotion, 9, 87-108.

Isen, A.M. (2000). Positive affect and decision making. In M. Lewis \& J.M. Havilan-Jones (Eds.), Handbook of Emotions ( $2^{\text {nd }}$ Ed.). New York: The Guilford Press.

Kahneman, D. (1973). Attention and effort. Prentice Hall: New Jersey.

Lang, P. J., Bradley, M. M., \& Cuthbert, B. N. (1997). International affective picture system (IAPS): Technical manual and affective ratings. NIMH Center for the Study of Emotion and Attention

Mayer, J. D. \& Gaschke, Y. N. (1988). The experience and metaexperience of mood. Journal of Personality and Social Psychology, 55, $102-111$

Macmillan, N. A. \& Creelman, C. D. (1991). Detection theory: A user's guide. New York: Cambridge University Press.

Niedenthal, P. M., Halberstadt, J. B., \& Setterlund, M. B. (1997). Being happy and seeing "happy": Emotional state mediates visual word recognition. Cognition and Emotion, 11, 403-432.

Raddatz, K.R., Uhlarik, J. \& Foura, J. E. (2004, September). Signal detection theory applied to a size comparison task. Proceedings of the $48^{\text {th }}$ Annual Meeting of the Human Factors and Ergonomics Society, New Orleans, LA.

Schmorrow, D., Stanney, K.M., Wilson, G., \& Young, P. (2006). Augmented cognition in human-system interaction. In G. Salvendy (Eds.), Handbook of Human Factors and Ergonomic ( $3^{\text {rd }}$ Ed.). New Jersey: Wiley and Sons, Inc.

Schneider, W., Eschman, A., \& Zuccolotto, A. (2002) E-Prime Reference Guide. Pittsburgh: Psychology Software Tools Inc.

Staal, M.A. (2004). Stress, cognition, and human performance: A literature review and conceptual framework. Technical Report NASA/TM2004-212824.

Tran, T.Q \& Raddatz, K.R, (2006, October). The effects of transient emotional state and workload on size scaling in perspective displays. Proceedings of the $50^{\text {th }}$ Annual Meeting of the Human Factors and Ergonomics Society, San Francisco, CA.

Wickens, C. D., Lee, J. D., Liu, Y., \& Gordon Becker, S. E.(2004) An Introduction to Human Factors Engineering $\left(2^{\text {nd }} \mathrm{Ed}\right.$.). New Jersey: Prentice Hall.

World Construction Set 4 [Computer software]. (1998). Brighton, CO: Questar Productions, LLC. 